SCIREA Journal of Mathematics

http://www.scirea.org/journal/Mathematics

January 20, 2022

Volume 7, Issue 1, February 2022

https://doi.org/10.54647/mathematics11304

\title{
Basic Nature of intermolecular attraction forces and Comparison with forces like Van Der Waals and hydrogen bonds by "Multi Molecule Theory"
}

\author{
Satyavarapu Naga Parameswara Gupta \\ Retired Assistant General Manager, Bhilai Steel Plant, Res 1B / Street 57 / Sector 8 / Bhilai \\ 490008, India \\ Email: snp.gupta@gmail.com
}

\begin{abstract}
:
This is a fundamental result. What is the basic nature the intermolecular attraction forces? These questions were not thought off earlier. All the earlier conclusions were oriented towards finding the value of inter molecular attraction forces. We took the nature of these forces to be Newtonian Gravitation attraction forces between individual molecules vectorially added for the total set of molecules under consideration. This method can be applied to any molecule or chemical which is having all the three states of matter. For an example, for proving this concept, we tried to explain the three states of water. Due to these attraction forces between the molecules, some pairs of molecules come near to each other and hit and bounce like rubber balls. We tried to detect these inter molecular hits. We wrote macros in Visual Basic for Excel to find these hits that happened in an iteration. We plotted addresses of colliding molecules in a designated area in the worksheet. We got another new finding. This pattern of hits is changing with inter molecular distances, by changing all the
\end{abstract}


inter molecular distances proportionally without changing the relative positions of molecules with each other. While checking with results this method with of states of water, we also will discuss about effect of Temperature. We will compare the effect of other forces like, Vander Waals (London dispersion forces) forces, hydrogen bonds, Keesom etc.. This method can explain Brownian motion etc.

Keywords: Gravitation, states of matter, hydrodynamics, molecular processes, molecular data, Multi Molecular Theory, SITA simulations, Dynamic Universe Model. Van Der Waals Forces, Singularity-free cosmology, Blue shifted Galaxies, Red shifted Galaxies, Grazing radiation frequency changes, Formation of Elements, Nucleosynthesis

\section{Intermolecular attraction forces:}

Let's see various types of intermolecular attraction forces segregated on the type of interaction. First Ion-ion type is seen in Ions only. The second Ion-Dipole types are seen between polar molecules and ions only. The third is Hydrogen bonding is seen in molecules that contained Nitrogen, Oxygen and Florin: link is shared Hydrogen atom. The fourth type Dipole-Dipole which is seen in Polar molecules only. The fifth type is Dipole - Induced Dipole, which can be observed in species where at least one molecule must be polar. And the sixth type is Keesom forces which is observed between Permanent Dipole -Permanent Dipole species. These types intermolecular forces are specifically observable in some particular species only one of the molecules must be a type of Dipole.

The Seventh type is Van Der Waals Forces (London dispersion forces), which can be observed in all types of molecules. It should be noted Van der Waals force also need induced Dipoles. We will discuss about this in 3.1 .

In this paper, we are working on a new type of intermolecular force that is applicable for all types and species of molecules and which will NOT involve any type of Intermolecular Ion or DIPOLE attractions. This inter molecular force is purely Newtonian Gravitation based and depends on the collective vectorial sum of attraction forces of mass of molecules only.

Let's go into details.... 


\subsection{SSMMT Formation:}

We took Dynamic Universe Model's SITA simulation software as it is, and we substituted its astronomical masses and distances values, with molecular masses and inter molecular distances values of water with other molecular values contaminations. This software is now supplemented with newly developed software for finding inter molecular HITs. We used this new set of SITA simulation software for using in this present platform.

We must mention that just "Newtonian Gravitation" as the force of attraction is NOT sufficient to give enough force between any single pair of Molecules. But the collective vectoral sum of these forces of Multiple molecules in SSMMT will do the trick.

Here in this paper, we will try to develop some equations for inter molecular forces, inter molecular hits which will give results like explaining the states of matter, and which will lead into Brownian motions, coefficient of diffusion etc., using this Savitri Subbarao Multi Molecule Theory (We call this SSMMT) instead of the age old 'Single Molecule theory'.

Let's calculate the resulting force vector due to Newtonian gravitation on a single molecule due to all the other molecules in the droplet. This calculation procedure is repeated for all the molecules in the droplet with the help of SSMMT.

\section{History:}

In 1959, Alder and Wainwright ${ }^{[4]}$ used an IBM 704 computer to simulate perfectly elastic collisions between hard spheres. They said " this many-body problem" was solved numerically using simultaneous equations of motion. Numerical methods give raise to errors always. This method solves many problems in both equilibrium and nonequilibrium statistical mechanics [5]. Probably the first simulation of matter, Gibson et al. simulated radiation damage of solid copper by using a Born-Mayer type of repulsive interaction along with a cohesive surface force. In his paper "Correlations in the Motion of Atoms in Liquid Argon" in 1964, Rehman A., said "The pair-correlation function and the constant of selfdiffusion are found to agree well with experiment; the latter is $15 \%$ lower than the experimental value. The spectrum of the velocity autocorrelation function shows a broad maximum in the frequency region $\omega=0.25\left(\mathrm{k}_{\mathrm{B}} \mathrm{T} / \hbar\right)$. He used a system of 864 particles interacting with a Lennard-Jones potential and obeying classical equations of motion [6]. These are some of the N-body problem type solutions about 60 years back. 
We could not find any information about the work of earlier physicists directly on the BASIC NATURE of the 'force of attraction between molecules' or 'state changes of water' of water (inter molecular attraction forces) in Google, Bing or Academia searches. But we did find some contributors that calculated the approximate value of this force.

\subsection{Van Der Waals Forces (London dispersion forces):}

When two molecules are near to each other polarisation will occur, due to the electrons in the molecule will be polarised and attracted towards another molecule which is oppositely polarised and with induced charges.

Van der Waals force is a distance-dependent interaction between atoms or molecules. These molecule-to-molecule attraction forces are not chemical electronic bonds like ionic or covalent bonds. They are comparatively weak and therefore more susceptible to disturbance. The van der Waals force quickly vanishes at longer distances between interacting molecules. If no other force is present, the distance between atoms at which the force becomes repulsive rather than attractive as the atoms approach one another is called the van der Waals contact distance; this phenomenon results from the mutual repulsion between the atoms' electron clouds as Described by Reginald H.; Grisham [7]

This is also an intermolecular force like [8] the London dispersion force between instantaneously induced dipoles and It is sometimes applied to the Debye force between a permanent dipole and a corresponding induced dipole or to the Keesom force between permanent molecular dipoles.

\section{The main characteristics of van der Waals forces are:[9]}

They are weaker than normal covalent and ionic bonds.

Van der Waals forces are additive and cannot be saturated.

They have no directional characteristic.

They are all short-range forces and hence only interactions between the nearest particles need to be considered (instead of all the particles).

Van der Waals attraction is greater if the molecules are closer.

Van der Waals forces are independent of temperature except dipole - dipole interactions. [10]

\subsection{Van der Waals forces between macroscopic objects--The microscopic theory:}


For macroscopic bodies with known volumes and numbers of atoms or molecules per unit volume, the total van der Waals force is often computed based on the "microscopic theory" as the sum over all interacting pairs. It is necessary to integrate over the total volume of the object, which makes the calculation dependent on the objects' shapes. For example, the van der Waals' interaction energy between spherical bodies of radii R1 and R2 and with smooth surfaces was approximated in 1937 by Hamaker [11] (using London's famous 1937 equation for the dispersion interaction energy between atoms/molecules [12] as the starting point) by:

$$
U\left(Z ; R_{1}, R_{2}\right)=-\frac{A}{6}\left(\frac{2 R_{1} R_{2}}{\left(z^{2}-\left(R_{1}+R_{2}\right)^{2}\right)}+\frac{2\left(R_{1} R_{2}\right)}{z^{2}-\left(R_{1}-R_{2}\right)^{2}}+\ln \left[\frac{z^{2}-\left(R_{1}+R_{2}\right)^{2}}{z^{2}-\left(R_{1}-R_{2}\right)^{2}}\right]\right)
$$

where $\mathrm{A}$ is the Hamaker coefficient, which is a constant $\left(\sim 10^{-19}-10^{-20} \mathrm{~J}\right)$ that depends on the material properties (it can be positive or negative in sign depending

on the intervening medium), and $\mathrm{z}$ is the center-to-center distance; i.e., the sum of $\boldsymbol{R}_{1}, \boldsymbol{R}_{2}$, and $\boldsymbol{r}$ (the distance between the surfaces): $z=\left(R_{1}+R_{2}+r\right)$.

In the limit of close-approach, the spheres are sufficiently large compared to the distance between them; i.e., $\boldsymbol{r}<<\boldsymbol{R}_{1}$ or $\boldsymbol{R}_{2}$, so that equation (1) for the potential

energy function simplifies to:

$$
U\left(r ; R_{1}, R_{2}\right)=-\frac{\left(A R_{1} R_{2}\right)}{6 r\left(R_{1}+R_{2}\right)}
$$

The van der Waals force between two spheres of constant radii $\left(\boldsymbol{R}_{1}\right.$ and $\boldsymbol{R}_{2}$ are treated as parameters) is then a function of separation since the force on an object is

the negative of the derivative of the potential energy function, . This yields:

$$
F_{v w}(r)=-\frac{\left(A R_{1} R_{2}\right)}{6 r\left(R_{1}+R_{2}\right)}
$$

The van der Waals forces between objects with other geometries using the Hamaker model have been published in the literature.[13]

From the expression above, it is seen that the van der Waals force decreases with decreasing size of bodies (R). Nevertheless, the strength of inertial forces, such as gravity and drag/lift, decrease to a greater extent. Consequently, the van der Waals forces become dominant for collections of very small particles such as very fine-grained dry powders (where there are no capillary forces present) even though the force of attraction is smaller in magnitude than it is for larger particles of the same substance. Such powders are said to be cohesive, meaning 
they are not as easily fluidized or pneumatically conveyed as their more coarse-grained counterparts. Generally, free-flow occurs with particles greater than about $250 \mu \mathrm{m}$. The van der Waals force of adhesion is also dependent on the surface topography. If there are surface asperities, or protuberances, that result in a greater total area of contact between two particles or between a particle and a wall, this increases the van der Waals force of attraction as well as the tendency for mechanical interlocking.

\section{The microscopic theory assumes pairwise additivity. It neglects many-body interactions} and retardation. A more rigorous approach accounting for these effects, called the "macroscopic theory" was developed by Lifshitz in 1956.[15] Langbein derived a much more cumbersome "exact" expression in 1970 for spherical bodies within the framework of the Lifshitz theory[16] while a simpler macroscopic model approximation had been made by Derjaguin as early as 1934.[17] Expressions for the van der Waals forces for many different geometries using the Lifshitz theory have likewise been published.

We have not used the above Microscopic theory

\section{Mathematical Background for SSMMT}

Let's take an example of $\mathrm{H}_{2} \mathrm{O}$, this example can be any chemical or Eutectic solution like steel....

Let us assume an inhomogeneous set of $\mathrm{N}$ Molecules as a colloid. This colloid is mix of water molecules, Individual proteins, polymers of living matter such as DNA, RNA, actin or microtubules, molecular motors etc. Water is universal solvent, universally available, so we take water + colloid particles for this theory. These don't react with each other chemically. All these molecules behave like bouncing rubber balls, sizes are different. All these set will have different masses in accordance with their type. This colloid will lead to have Brownian motions of the Nano-Particles due to mutual gravitation attraction forces between individual particles. Let's take total number of molecules to be 133, to use SITA calculations. We call these calculations as SSMMT.

We will start with133 particles / molecules in a Micro-cubical cubical in a glass beaker. We will assume a Micro-Cubicle in this beaker with invisible elastic walls. We will use 3D Cartesian coordinates with some appropriate center of its axes in this beaker. 
- All the boundaries are perfectly elastic. Any Nano-Particle hitting the boundary will return according to Newtons laws. We will take nano distances and pico-second times as appropriate. To this case. We assume all the molecules to be perfect elastic spheres. There will be bumping and collisions between particles, and each pair will move and trace their path back after the elastic collision between the two. We can detect collisions by SSMMT software by two bumping particles when the center-to-center distance is less than or equal to the sum of two molecular radii of these Nano-spheres.

So, it is obvious that the distance between the two molecules will increase and their velocities reverses after each pair's collision.

Initially, let us assume a set of $\mathrm{N}$ mutually gravitating point masses in a system under Newtonian Gravitation. All these Nano-particles will have some finite radii $r_{i}$.

In this paper we will not consider gravitational repulsion at very low distances, only the bumping will happen at that distance, later we will introduce this concept in a next paper....

\section{Similarly, we will introduce the Viscosity forces in another forthcoming paper.}

Initially, let us assume a set of $\mathrm{N}$ mutually gravitating point masses in a system under Newtonian Gravitation. All these Nanobio-particles will have some finite radii $r_{i}$ which we will use for the calculation of bumping or collision in the SSMMT software.

Let the $\alpha^{\text {th }}$ point mass has mass $\mathrm{m}_{\alpha}$, and is in position $\mathrm{x}_{\alpha}$. In addition to the mutual gravitational force, there exists an external $\phi$ ext, due to other molecules which also influence the total force $\mathrm{F}_{\alpha}$ acting on the point mass $\alpha$. In this case, the $\phi_{\text {ext }}$ is not a constant universal Gravitational field but it is the total vectorial sum of fields at $\mathrm{x}_{\alpha}$ due to all the external to its system bodies and with that configuration at that moment of time.

Total Mass of system $=\sum_{\alpha=1}^{N} m_{\alpha}$

Total force on the point mass $\alpha$ is $F \alpha$, Let $F_{\alpha \beta}$ is the gravitational force on the $\alpha^{\text {th }}$ point mass due to $\beta^{\text {th }}$ point mass.

$$
F_{\alpha}=\sum_{\substack{\alpha=1 \\ \alpha \neq \beta}}^{N} F_{\alpha \beta}-m_{\alpha} \nabla_{\alpha} \Phi_{e x t}(\alpha)
$$


Moment of inertia tensor (We will assume moment inertia is the dominating, but for theoretical calculations for this paper we will ignore Viscus forces)

Consider a system of $\mathrm{N}$ point masses with mass $\mathrm{m}_{\alpha}$, at positions $\mathrm{X}_{\alpha}, \alpha=1,2, \ldots \mathrm{N}$; The moment of inertia tensor is in external back ground field $\phi_{\text {ext. }}$.

$$
I_{j k}=\sum_{\alpha=1}^{N} m_{\alpha} x_{j}^{\alpha} x_{k}^{\alpha}
$$

Its second derivative is

$$
\frac{d^{2} I_{j k}}{d t^{2}}=\sum_{\alpha=1}^{N} m_{\alpha}\left(x_{j}^{\alpha} x_{k}^{\alpha}+x_{j}^{\alpha} \dot{\circ}_{k}^{\alpha}+x_{j}^{\alpha} x_{k}^{\alpha}\right)
$$

The total force acting on the point mass $\alpha$ is and $F$ is the unit vector of force at that place of that component.

$$
F_{j}^{\alpha}=m_{\alpha} x_{j}^{\alpha}=\sum_{\substack{\beta=1 \\ \alpha \neq \beta}}^{N} \frac{G m_{\alpha} m_{\beta}\left(x_{j}^{\beta}-x_{j}^{\alpha}\right) \hat{F}}{\left|x^{\beta}-x^{\alpha}\right|^{3}}-\nabla \Phi_{e x t, j} m_{\alpha}
$$

Writing a similar formula for $\mathrm{F}^{\alpha} \mathrm{k}$

$$
F_{k}^{\alpha}=m_{\alpha} x_{k}^{\alpha}=\sum_{\substack{\beta=1 \\ \alpha \neq \beta}}^{N} \frac{G m_{\alpha} m_{\beta}\left(x_{k}^{\beta}-x_{k}^{\alpha}\right) \hat{F}}{\left|x^{\beta}-x^{\alpha}\right|^{3}}-\nabla \Phi_{e x t, k} m_{\alpha}
$$

$\mathrm{OR}=>$

$$
x_{j}^{\alpha}=\sum_{\substack{\beta=1 \\ \alpha \neq \beta}}^{N} \frac{G m_{\beta}\left(x_{j}^{\beta}-x_{j}^{\alpha}\right) \hat{F}}{\left|x^{\beta}-x^{\alpha}\right|^{3}}-\nabla \Phi_{e x t}
$$

And $=>$

$$
x_{k}^{\alpha}=\sum_{\substack{\beta=1 \\ \alpha \neq \beta}}^{N} \frac{G m_{\beta}\left(x_{k}^{\beta}-x_{k}^{\alpha}\right)}{\left|x^{\beta}-x^{\alpha}\right|^{3}}-\nabla \Phi_{e x t}
$$

Lets define Energy tensor ( in the external field $\phi_{\text {ext }}$ ) 


$$
\begin{aligned}
\frac{d^{2} I_{j k}}{d t^{2}}= & 2 \sum_{\alpha=1}^{N} m_{\alpha}\left(x_{j}^{\alpha} \dot{x}_{k}^{\alpha}\right)+\sum_{\substack{\alpha=1 \\
\alpha \neq \beta}}^{N} \sum_{\substack{\beta=1 \\
\alpha \neq \beta}}^{N} \frac{G m_{\alpha} m_{\beta}\left\{\left(x_{k}^{\beta}-x_{k}^{\alpha}\right) x_{j}^{\alpha}+\left(x_{j}^{\beta}-x_{j}^{\alpha}\right) x_{k}^{\alpha}\right\}}{\left|x^{\beta}-x^{\alpha}\right|^{3}} \\
& -\sum_{\alpha=1}^{N} \nabla \Phi_{e x t} m_{\alpha} x_{j}^{\alpha}-\sum_{\alpha=1}^{N} \nabla \Phi_{e x t} m_{\alpha} x_{k}^{\alpha}
\end{aligned}
$$

Lets denote Potential energy tensor $=\mathrm{Wjk}=$

$$
\sum_{\substack{\alpha=1 \\ \alpha \neq \beta}}^{N} \sum_{\substack{\beta=1 \\ \alpha \neq \beta}}^{N} \frac{G m_{\alpha} m_{\beta}\left\{\left(x_{k}^{\beta}-x_{k}^{\alpha}\right) x_{j}^{\alpha}+\left(x_{j}^{\beta}-x_{j}^{\alpha}\right) x_{k}^{\alpha}\right\}}{\left|x^{\beta}-x^{\alpha}\right|^{3}}
$$

Lets denote Kinetic energy tensor $=2 \mathrm{~K}_{\mathrm{jk}}=2 \sum_{\alpha=1}^{N} m_{\alpha}\left(\begin{array}{c}\dot{0}^{\alpha} \\ x_{j}^{\alpha} \\ x_{k}^{\alpha}\end{array}\right)$

Lets denote External potential energy tensor $=2 \Phi_{\mathrm{jk}}$

$$
=\sum_{\alpha=1}^{N} \nabla \Phi_{e x t} m_{\alpha} x_{j}^{\alpha}+\sum_{\alpha=1}^{N} \nabla \Phi_{e x t} m_{\alpha} x_{k}^{\alpha}
$$

Hence $\frac{d^{2} I_{j k}}{d t^{2}}=W_{j k}+2 K_{j k}-2 \Phi_{j k}$

Here in this case

$$
\begin{aligned}
& F(\alpha)=\sum_{\substack{\beta=1 \\
\alpha \neq \beta}}^{N} F_{\alpha \beta}-\nabla_{\alpha} \Phi_{e x t}(\alpha) m_{\alpha} \\
& =\sum_{\substack{\beta=1 \\
\alpha \neq \beta}}^{N} \frac{G m_{\alpha} m_{\beta}\left(x^{\beta}-x^{\alpha}\right)}{\left|x^{\beta}-x^{\alpha}\right|^{3}}-\nabla \Phi_{e x t} m_{\alpha} \\
& =\left\{x(\text { int })-\nabla_{\alpha} \Phi_{e x t}(\alpha)\right\} m_{\alpha} \\
& \stackrel{x}{x}(\alpha)=\sum_{\substack{\beta=1 \\
\alpha \neq \beta}}^{N} \frac{G m_{\beta}\left(x^{\beta}-x^{\alpha}\right)}{\left|x^{\beta}-x^{\alpha}\right|^{3}}-\nabla \Phi_{e x t}
\end{aligned}
$$


We know that the total force at $x(\alpha)=F_{t o t}(\alpha)=-\nabla_{\alpha} \Phi_{t o t}(\alpha) m_{\alpha}$ Total PE at $\alpha=m_{\alpha} \Phi_{\text {tot }}(\alpha)=-\int F_{\text {tot }}(\alpha) d x$

$$
\begin{aligned}
& =-\int\left\{\sum_{\substack{\beta=1 \\
\alpha \neq \beta}}^{N} x_{\text {int }}^{\circ \alpha} m_{\alpha}-\nabla_{\alpha} \Phi_{\text {ext }}(\alpha) m_{\alpha}-\right\} d x \\
& =\int \sum_{\substack{\beta=1 \\
\alpha \neq \beta}}^{N} \frac{G m_{\beta} m_{\alpha}\left(x^{\beta}-x^{\alpha}\right)}{\left|x^{\beta}-x^{\alpha}\right|^{3}} d x-\int \nabla \Phi_{e x t} m_{\alpha} d x
\end{aligned}
$$

Therefore total Gravitational potential $\phi_{\text {tot }}(\alpha)$ at $\mathrm{x}(\alpha)$ per unit mass

$$
\Phi_{t o t}(\alpha)=\Phi_{e x t}-\sum_{\substack{\beta=1 \\ \alpha \neq \beta}}^{N} \frac{G m_{\beta}}{x^{\beta}-x^{\alpha} \mid}
$$

\section{Lets discuss the properties of $\phi_{\text {ext }}$ :-}

$\phi_{\text {ext }}$ can be subdivided into 3 parts mainly

$\phi_{\text {ext }}$ due to higher level system, $\phi_{\text {ext }}$-due to lower level system, $\phi_{\text {ext }}$ due to present level. [ Level : when we are considering point masses in the same system (Galaxy), they are at the same level, a higher level for a cluster of galaxies, and a lower level is for planets \& asteroids].

$\phi_{\text {ext }}$ is due to lower levels : If the lower level is existing, at the lower level of the system under consideration, then its own level was considered by system equations. If this lower level exists anywhere outside of the system, the center of (mass) gravity outside systems (Galaxies) will act as (unit) its own internal lower level, practically will be considered into calculations. Hence separate consideration of any lower level is not necessary.

\section{SYSTEM - ENSEMBLE:}

Until now we have considered the system level equations and the meaning of $\phi_{\text {ext. }}$ Now let's consider an ENSEMBLE of system consisting of $\mathrm{N}_{1}, \mathrm{~N}_{2} \ldots$ Njpoint masses in each. These systems are moving in the ensemble due to mutual gravitation between them. For example, 
each system is a Galaxy, and then ensemble represents a local group. Suppose number of Galaxies is j, Galaxies are systems with point masses N1, N2 ....NJ, we will consider $\phi_{\text {ext }}$ as discussed above. That is we will consider the effect of only higher level system like external Galaxies as a whole, or external local groups as a whole.

Ensemble Equations (Ensemble consists of many systems)

$$
\frac{d^{2} I^{\gamma}{ }_{j k}}{d t^{2}}=W_{j k}^{\gamma}+2 K_{j k}^{\gamma}-2 \Phi_{j k}^{\gamma}
$$

Here ${ }^{\gamma}$ denotes Ensemble.

This $\Phi^{\gamma} \mathrm{jk}$ is the external field produced at system level. And for system

$$
\frac{d^{2} I_{j k}}{d t^{2}}=W_{j k}+2 K_{j k}-2 \Phi_{j k}
$$

Assume ensemble in a isolated place. Gravitational potential $\phi_{\text {ext }}(\alpha)$ produced at system level is produced by Ensemble and $\quad \phi^{\gamma} \mathrm{ext}(\alpha)=0$ as ensemble is in a isolated place.

$$
\Phi_{t o t}^{\gamma}(\alpha)=\Phi_{e x t}^{\gamma}-\sum_{\substack{\beta=1 \\ \alpha \neq \beta}}^{N^{\gamma}} \frac{G m_{\beta}^{\gamma}}{\left|x^{\gamma \beta}-x^{\gamma \alpha}\right|}
$$

As Ensemble situated in an isolated place, Gravitational potential $\phi^{\gamma_{\text {ext }}}(\alpha)=0$

Therefore

$$
\Phi_{t o t}^{\gamma}=\Phi_{e x t}(\alpha)=-\sum_{\substack{\beta=1 \\ \alpha \neq \beta}}^{N^{\gamma}} \frac{G m_{\beta}^{\gamma}}{\left|x^{\gamma \beta}-x^{\gamma \alpha}\right|}
$$

And

$$
2 \Phi_{j k}=-\frac{d^{2} I_{j k}}{d t^{2}}+W_{j k}+2 K_{j k}
$$

$$
=\sum_{\alpha=1}^{N} \nabla \Phi_{e x t} m_{\alpha} x_{j}^{\alpha}+\sum_{\alpha=1}^{N} \nabla \Phi_{e x t} m_{\alpha} x_{k}^{\alpha}
$$

\section{AGGREGATE Equations(Aggregate consists of many Ensembles )}




$$
\frac{d^{2} I_{j k}^{\delta \gamma}}{d t^{2}}=W_{j k}^{\delta \gamma}+2 K_{j k}^{\delta \gamma}-2 \Phi_{j k}^{\delta \gamma}
$$

Here $^{\delta}$ denotes Aggregate.

This $\Phi^{\delta \gamma j k}$ is the external field produced at Ensemble level. And for Ensemble

$$
\frac{d^{2} I^{\gamma}{ }_{j k}}{d t^{2}}=W_{j k}^{\gamma}+2 K_{j k}^{\gamma}-2 \Phi_{j k}^{\gamma}
$$

Assume Aggregate in an isolated place. Gravitational potential $\phi_{\text {ext }}(\alpha)$ produced at Ensemble level is produced by Aggregate and $\phi^{\delta \gamma_{\mathrm{ext}}}(\alpha)=0$ as Aggregate is in a isolated place.

Therefore

$$
\Phi_{t o t}^{\delta \gamma}(\alpha)=\Phi_{e x t}^{\delta \gamma}-\sum_{\substack{\beta=1 \\ \alpha \neq \beta}}^{N^{\delta \gamma}} \frac{G m_{\beta}^{\delta \gamma}}{\left|x^{\delta \gamma \beta}-x^{\delta \gamma \alpha}\right|}
$$

And

$$
\Phi_{j k}^{\gamma}=\sum_{\alpha=1}^{N^{\gamma}} \nabla \Phi_{e x t}^{\delta} m_{\alpha} x_{j}^{\delta \alpha}+\sum_{\alpha=1}^{N} \nabla \Phi_{e x t}^{\delta} m_{\alpha} x_{k}^{\delta \alpha}
$$

Total AGGREGATE Equations :( Aggregate consists of many Ensembles and systems)

Assuming these forces are conservative, we can find the resultant force by adding separate forces vectorially from equations (20) and (23).

$$
\Phi_{e x t}(\alpha)=-\sum_{\substack{\beta=1 \\ \alpha \neq \beta}}^{N^{\gamma}} \frac{G m_{\beta}^{\gamma}}{x^{\gamma \beta}-x^{\gamma \alpha} \mid}-\sum_{\substack{\beta=1 \\ \alpha \neq \beta}}^{N^{\delta \gamma}} \frac{G m_{\beta}^{\delta \gamma}}{x^{\delta \gamma \beta}-x^{\delta \gamma \alpha} \mid}
$$

This concept can be extended to still higher levels in a similar way.

\section{Corollary 1:}

$$
\frac{d^{2} I_{j k}}{d t^{2}}=W_{j k}+2 K_{j k}-2 \Phi_{j k}
$$


The above equation becomes a scalar Virial theorem in the absence of an external field, that is $\phi=0$ and is in a "steady state,"

$$
\text { i.e. } \begin{aligned}
\frac{d^{2} I_{j k}}{d t^{2}} & =0 \\
2 \mathrm{~K}+\mathrm{W} & =0
\end{aligned}
$$

But when the $\mathrm{N}$-bodies are moving under the influence of mutual gravitation without external field then only the above equation (28) is applicable.

\section{Corollary 2:}

Ensemble achieved a steady state,

$$
\begin{gathered}
\frac{d^{2} I_{j k}^{\gamma}}{d t^{2}}=0 \\
\text { i.e. } \\
W_{j k}^{\gamma}+2 K_{j k}^{\gamma}=2 \Phi_{j k}^{\gamma}
\end{gathered}
$$

This $\Phi \mathrm{jk}$ external field produced at system level. Ensemble achieved a steady state; means system also reached steady state.

$$
\begin{gathered}
\frac{d^{2} I_{j k}}{d t^{2}}=0 \\
\text { i.e. } \\
W_{j k}+2 K_{j k}=2 \Phi_{j k}^{\gamma}
\end{gathered}
$$

Equation (20) gives $\phi^{\gamma_{\text {tot }}}(\alpha)$, that is external potential field present at the system level . Combining Eqn (31) and eqn (9).

$$
2 \Phi_{e x t} j k=\sum_{\alpha=1}^{N} \nabla \Phi_{e x t} m_{\alpha} x_{j}^{\alpha}+\sum_{\alpha=1}^{N} \nabla \Phi_{e x t} m_{\alpha} x_{k}^{\alpha}
$$

The Equation 25 is the main powerful equation, which gives many results that are not possible otherwise today. This tensor can be subdivided into 21000 small equations without any differential equations or integral equations. Hence, this set up gives a unique solution of Cartesian X, Y, Z components of coordinates, velocities and accelerations of each point mass in the setup for that particular instant of time. instant of time. A point to be noted here is that 
the Dynamic Universe Model never reduces to General relativity on any condition. It uses a different type of mathematics based on Newtonian physics. This mathematics used here is relatively simple and straightforward. For all the mathematics, and the Excel based software, details are explained in the three books published by the author $[14,15,16]$

A point to be noted here is that the Dynamic Universe Model never reduces to General relativity on any condition. It uses a different type of mathematics based on Newtonian physics. This mathematics used here is simple and straightforward. As there are no differential equations present in Dynamic Universe Model, the set of equations give single solution in $\mathrm{x} \mathrm{y} \mathrm{z}$ Cartesian coordinates for every point mass for every time step. All the mathematics and the Excel based software details are explained in the three books published by the author[14, 15, 16] In the first book, the solution to N-body problem-called Dynamic Universe Model (SITA) is presented; which is singularity-free, inter-body collision free and dynamically stable. The Basic Theory of Dynamic Universe Model published in 2010 [14]. The second book in the series describes the SITA software in EXCEL emphasizing the singularity free portions. This book written in 2011 [15] explains more than 21,000 different equations. The third book describes the SITA software in EXCEL in the accompanying CD / DVD emphasizing mainly HANDS ON usage of a simplified version in an easy way. The third book is a simplified version and contains explanation for 3000 equations instead of earlier 21000 and this book also was written in 2011[16]. Some of the other papers published by the author are available at refs. $[3,5,8,9,10,11,17,19]$.

SITA solution can be used in many places like presently unsolved applications like Pioneer anomaly at the Solar system level, Missing mass due to Star circular velocities and Galaxy disk formation at Galaxy level etc. Here we are using it for prediction of blue shifted Galaxies.

We applied above theory and developed an Excel sheet. See the data availability statement for the fully functional Excel sheet referenced just before the References in this paper. In addition, all the screen captures shown in this paper were also were taken from the same sheet.

\section{Applying SSMMT to $\mathrm{H}_{2} \mathrm{O}$ and Screen shots:}

What factor changes with change of state of $\mathrm{H}_{2} \mathrm{O}$ ? Of course, temperature is the common thinking. Temperature is not basic nature the inter molecular attraction forces, but changes 
inter-molecular agitations and distances. How temperature is related to this method, which is more fundamental are also discussed here. We plunged into a more fundamental aspect i.e., inter molecular attraction forces and intermolecular distances... We took a distance factor $(D F)=1$ which represents inter molecular distances in a drop of contaminated water. And keeping the original molecular structure same changed all the inter molecular distances proportionally with different values of DFs... We took screen shots of that area for that value of DF value and shown these plots Inter Molecular Hits in this paper. We observe that number of inter molecule hits is decreasing from all the pairs hitting each other to no hitting pair depending on inter molecular distances. We can visualize very clearly for DF 0.02 to 0.08 the state will be ice, for DF 9 to 300 the state will be water and for DF 5000 to 10000 the state will be vapour. There are many MIXED intermediate states.

Water has polar O-H bonds called a hydrogen bonds. Since water has hydrogen bonds, it also has dipole-induced dipole and London dispersion forces. We will discuss these in detail in next section

\subsection{TEMPERATURE:}

Now let's see what happens with increase or decrease of temperature. The agitations of molecules will increase or decrease. So, what are these agitations? The molecules which are moving randomly will move more rapidly or less rapidly. Change of state will happen when the required energy is given or taken out. Now let's see in a little detail below. Full explanation with required proofs will be presented in our next paper.

\subsection{TEMPERATURE Increase:}

When we increase temperature the energy to molecules increases. That means they move with more velocities in the same directions in which they are moving before increase of temperature. Inter molecular hits will increase. Hence molecular agitations will increase. Inter molecular distances will increase. DF the distance factor will increase. From here we can go into our discussion in this paper.

\subsection{TEMPERATURE decrease:}

When we decrease temperature the energy to molecules decreases. That means they move with less velocities in the same directions in which they are moving before decrease of temperature. Inter molecular hits will decrease. Hence molecular agitations will decrease. Inter molecular distances will decrease. DF the distance factor will decrease. From here we can go into our discussion in this paper. 


\section{Inter Molecular Distances, Inter Molecular Hits and Distance Factor (DF):}

Let's take the colloid case in SSMMT calculations as discussed in the above Mathematical background section. We allow some iterations of calculations to happen. In every iteration of calculations, the positions of molecules will change. This happens due to the movements of all the molecules due to mutual Newtonian gravitational attraction forces. We can take any iteration result for our further analysis purposes. This iteration should be a stable one after sone initial disturbances. This iteration can be any particular one. No separate screening or selection process is required.

What we will do now is to change all these inter molecular distances proportionally in accordance with a Distance Factor. We will take all the set of distances as defined in the colloid as for a Distance factor 1 or simply $\mathbf{D F}=\mathbf{1}$. Then we will multiply this set of inter molecular distances with the DF whose value we will change manually from 0.02 to 90000 . With every value of DF, we will plot addresses of hitting molecule- pairs in a designated range in Excel of 133 Row X 133 columns. We named this range as "Inter_mol_Hits". We will store the address of hitting pair of molecules in the appropriate address location in that range.

Then we will collect a screen capture 133 Row X 133 columns after zooming out appropriately to fit in a single screen for that DF value. All these screen shots are presented below after trimming the unnecessary portions. All these are depicted in next section below....

\subsection{Screen Captures for different values of DFs:}

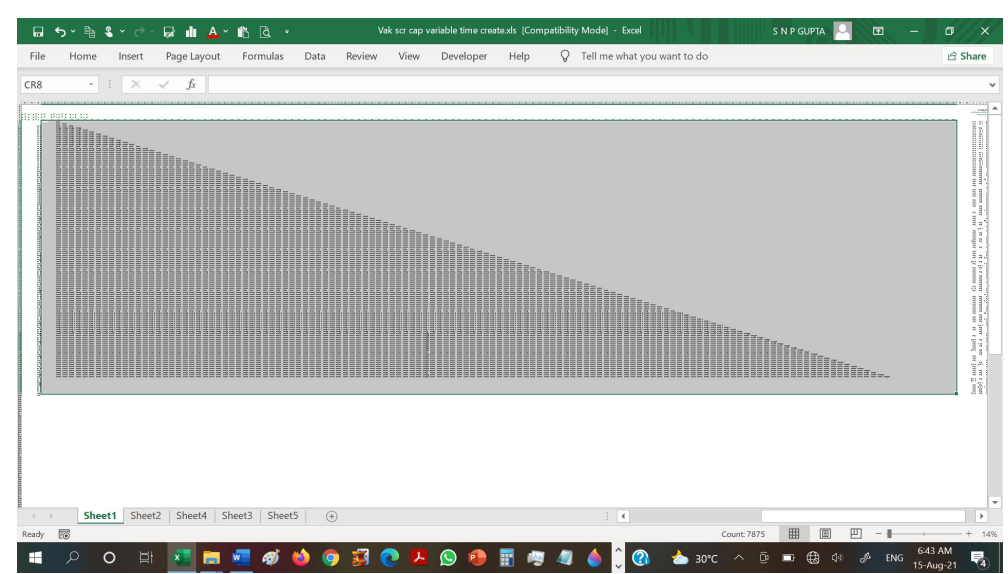


Fig 1. A sample screen capture can be seen here with all the inter molecular hits. If you enlarge this capture picture, you will find lots of rectangular dots, which are made up of some letters. Each of such letter combination indicates the address of two molecules which hit together or such molecules which had a hit together and are going apart after the hit. Here in this capture the background of the triangular pattern was shown as grey colour to have clear view of total range under consideration. All the later captures were snipped to grey colour area only to show all the inter molecular hits happened in that particular situation of DF value.

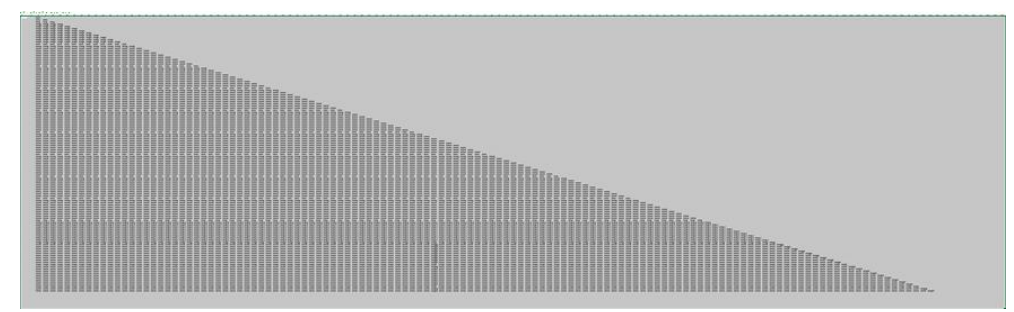

Fig 2. Above are Ice states for sure as all columns are full, i.e., DF 0.02 to 0.08

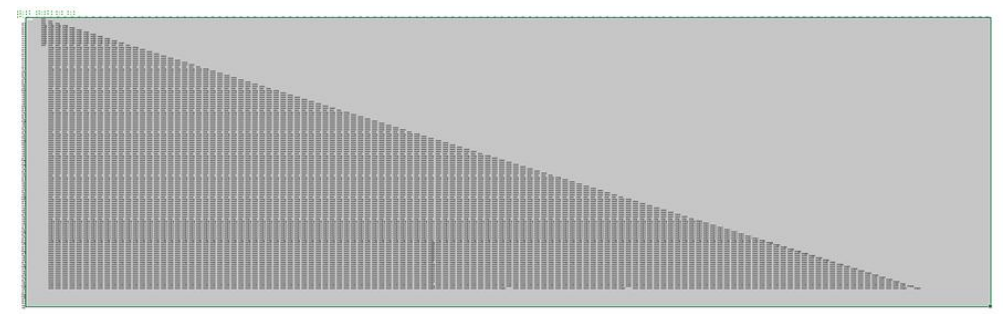

Fig 3.1. This screen shot is for $\mathrm{DF}=0.09$. We see Blanks in col CT, and in Col CU Blanks from 20 to 132 rows. Please note that in the $132^{\text {nd }}$ row a single blank is located in three columns viz., FH FY HM. We will use Excel method of showing the column numbers. As the memory length is limited, we made these figures small. This fig can be enlarged to see the full details.

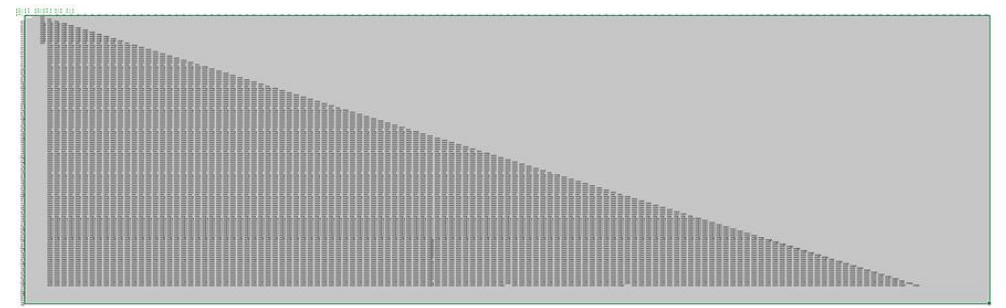

Fig 3.2. This screen shot is for $\mathrm{DF}=0.1$, Exactly same as above for $\mathbf{D F}=0.09$. We see Blanks in col CT, and in Col CU Blanks from 20 to 132 rows. Please note that in the $132^{\text {nd }}$ row a single blank is located in three columns viz., FH FY HM. We will use Excel method 
of showing the column numbers. As the memory length is limited, we made these figures small. This fig can be enlarged to see the full details.

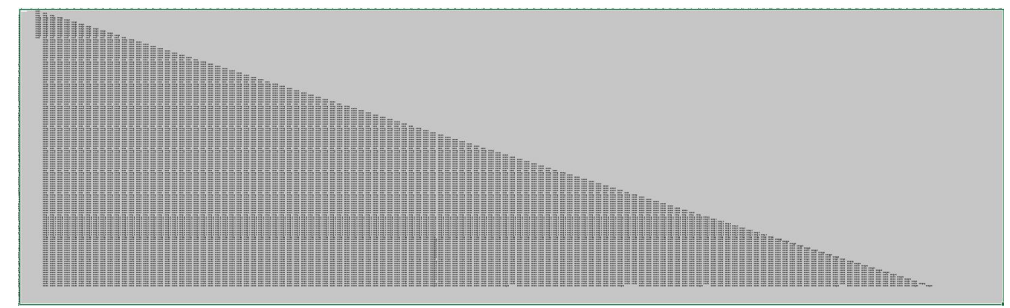

Fig 3.3. This screen shot is for $\mathrm{DF}=0.11$. We see Blanks in col CT, and in Col CU Blanks from 20 to 132 rows. Please note that in the $132^{\text {nd }}$ row a single blank is located in five columns viz., FH, FX, FY, GI, HB \& HM.

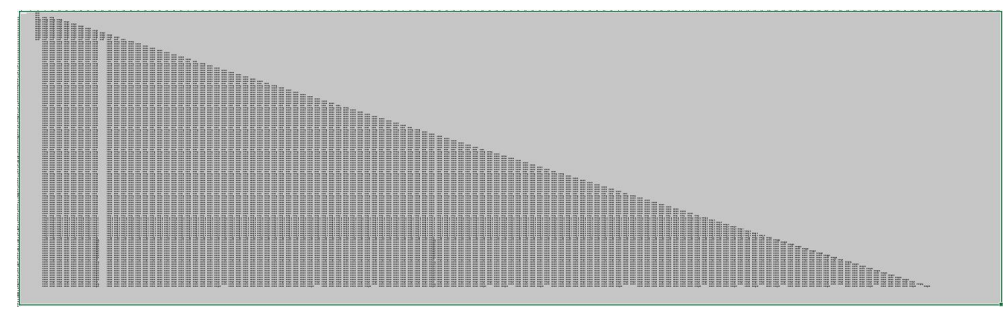

Fig 3.4. This screen shot is for $\mathrm{DF}=0.12$. We see Blanks in col CT, and in Columns CU \& DC Blanks from 20 to 132 rows. Please note that in the $132^{\text {nd }}$ row a single blank is located in eleven columns viz., FE, FH, FM, FX, FY, GI, GN, GT, HB, HG \& HM.

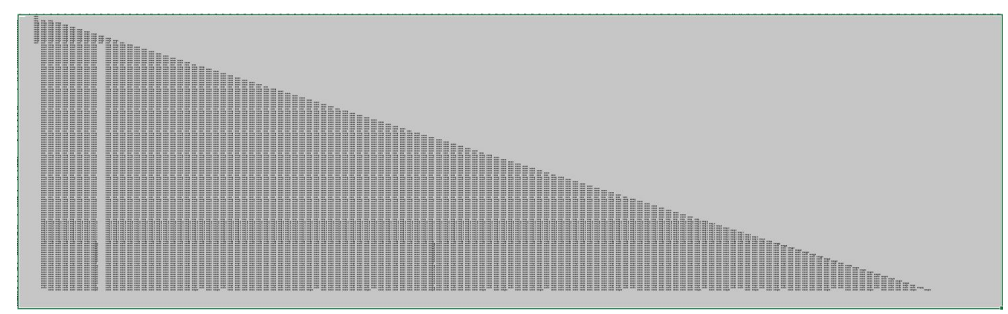

Fig 3.5. This screen shot is for DF= 0.13. We see Blanks in col CT, and in Columns CU \& DC Blanks from 20 to 132 rows. Please note that in the $132^{\text {nd }}$ row a single blank is located in 132 blank 19 columns.

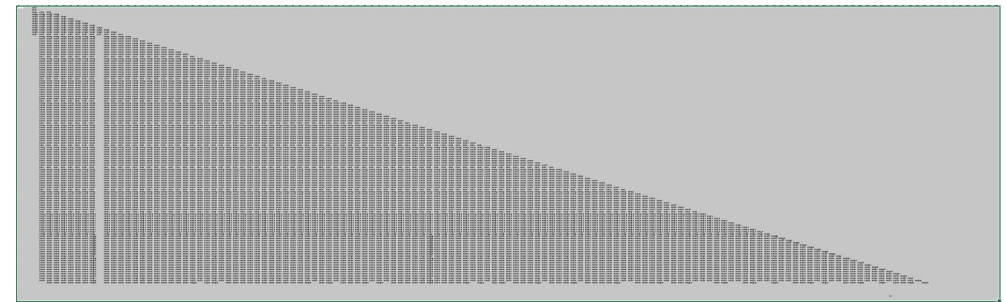


Fig 3.6. This screen shot is for $\mathrm{DF}=0.15$. We see Blanks in col CT, and in Columns CU \& DC Blanks from 20 to 132 rows. Please note that in the $132^{\text {nd }}$ row a single blank is located in 132 blank 32 columns.

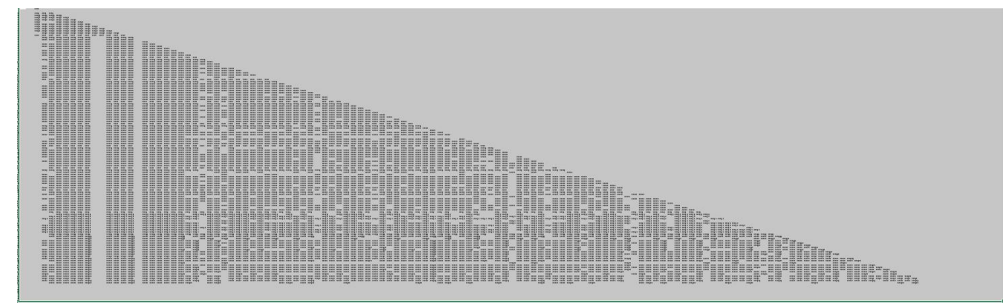

Fig 4.1. This screen shot is for $\mathrm{DF}=0.3$. We see Blanks in col $\mathrm{CT}$, and in Columns CU \& DB DC Blanks from 20 to 132 rows. Please note that many full column hits and partial column hits.

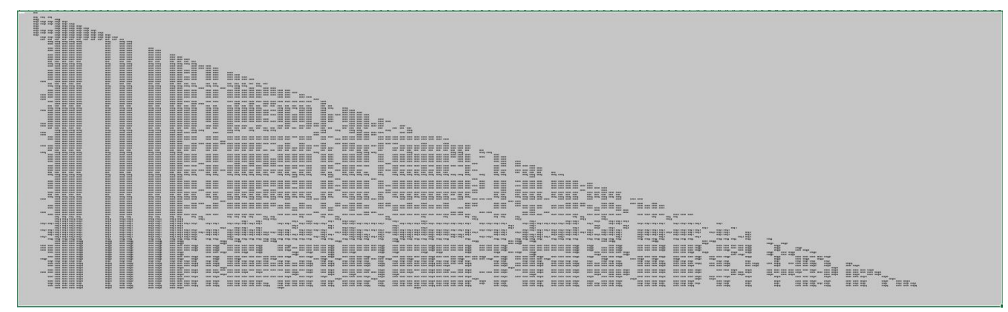

Fig 4.2. This screen shot is for $D F=0.45$. We see 4 Full Column hits and many partial column hits.

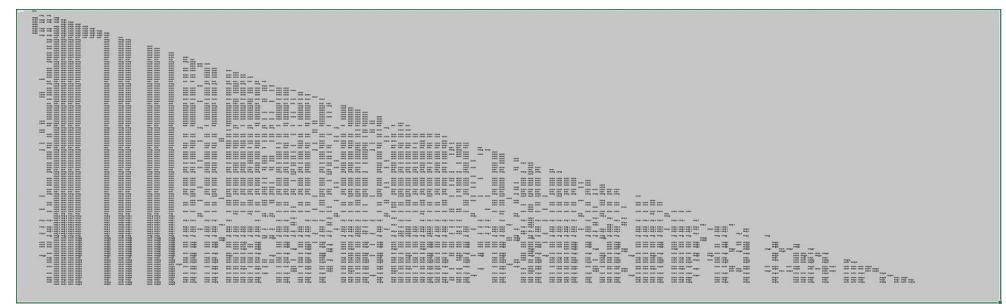

Fig 4.3. This screen shot is for $D F=0.5$. We see 10 Full column hits and many partial column hits.

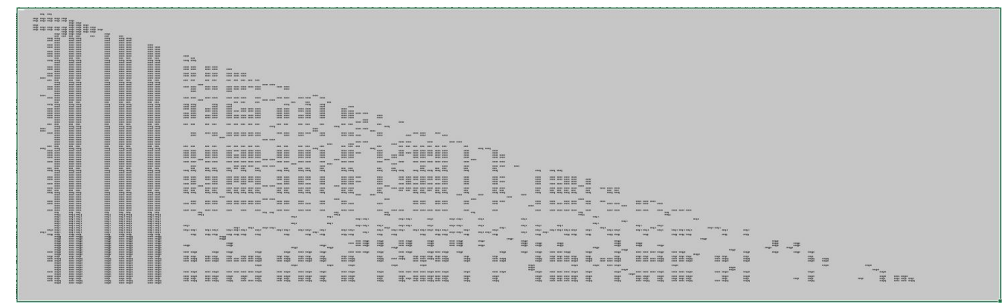

Fig 5.1. This screen shot is for $D F=0.9$. We see 8 Full column hits and many partial column hits.

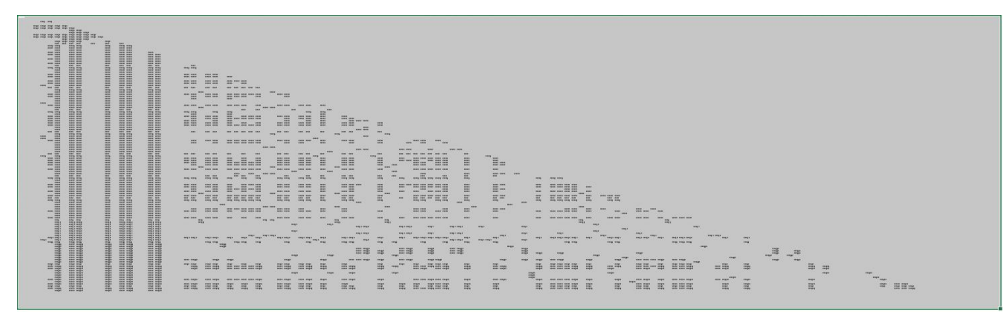


Fig 5.2. This screen shot is for $D F=1$. We see 8 Full column hits and many partial column hits.

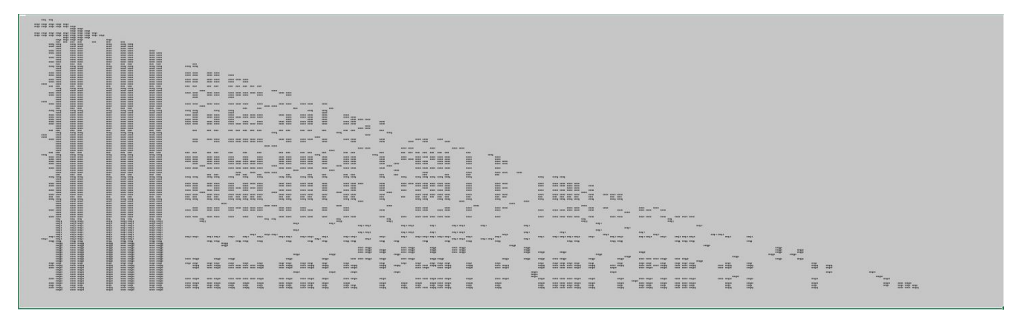

Fig 5.3. This screen shot is for $D F=1.1$. We see 8 Full column hits and many partial column hits.

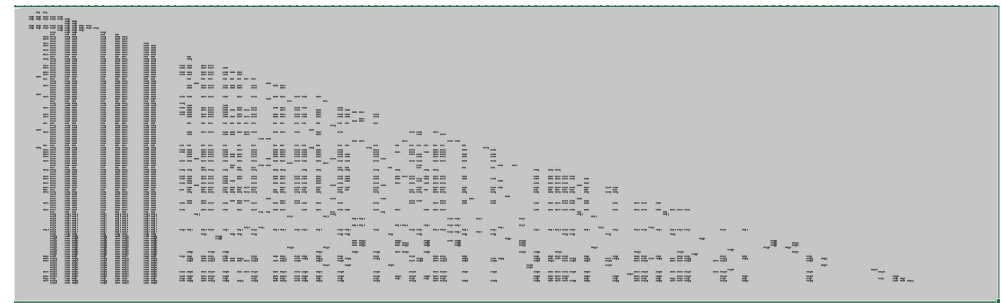

Fig 5.4. This screen shot is for $D F=1.2$. We see 8 Full column hits and many partial column hits.

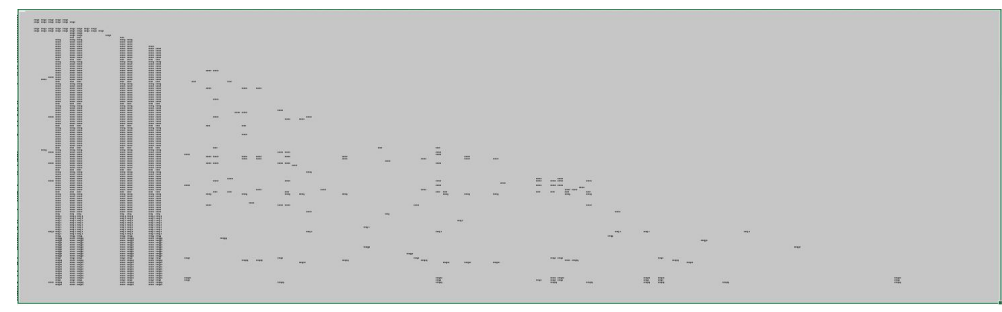

Fig 6.1. This screen shot is for $D F=3$. We see 7 Full column hits and many scattered hits.

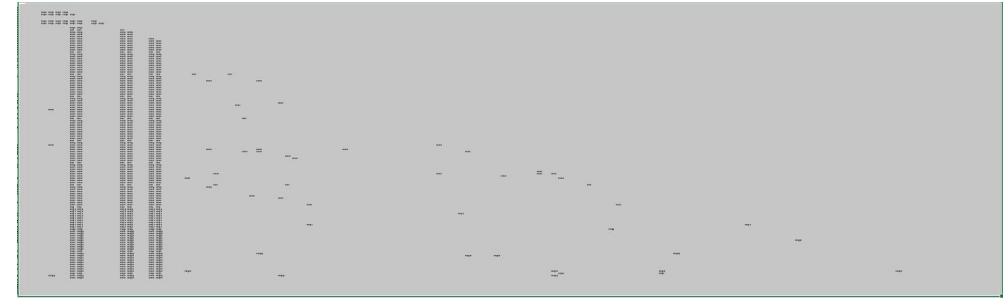

Fig 6.2. This screen shot is for $\mathrm{DF}=4.5$. We see 7 Full column hits and 50 scattered hits.

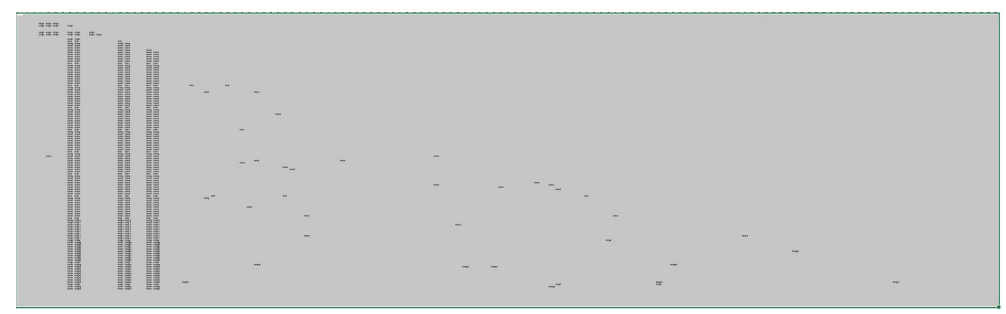

Fig 6.3. This screen shot is for $\mathrm{DF}=5$. We see 6 Full column hits and low scattered hits. 


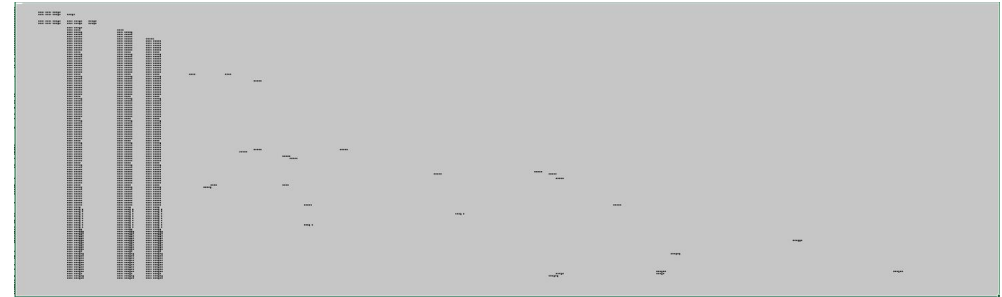

Fig 6.4. This screen shot is for $D F=6$. We see 6 Full column hits and 14 hits + some scattered hits.

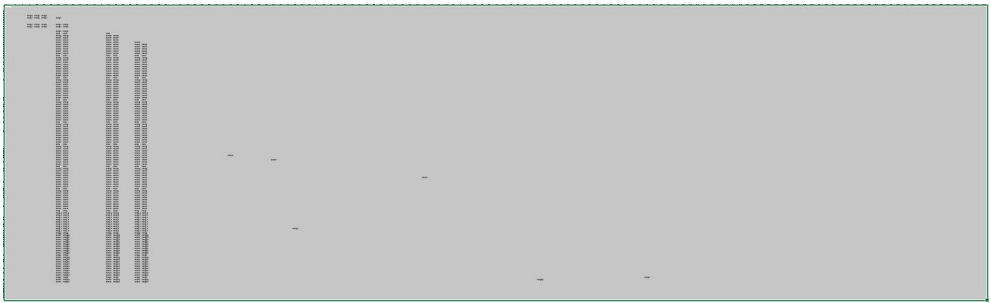

Fig 7.1. This screen shot is for $D F=9$. We see 6 Full column hits and 18 hits.

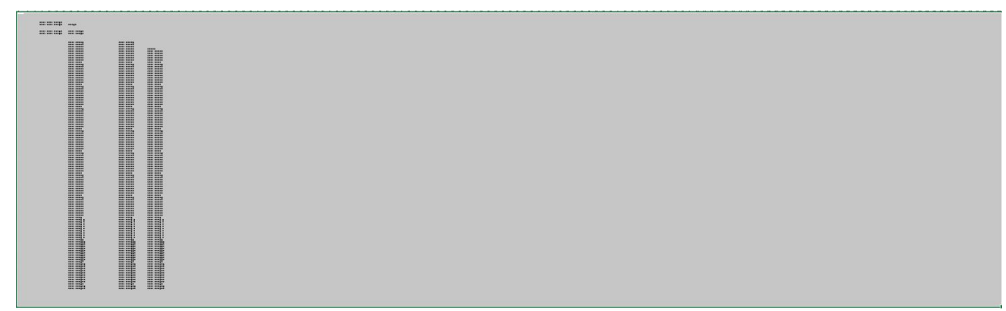

Fig 7.2. This screen shot is for $D F=30$. We see 6 Full column hits and 12 hits.

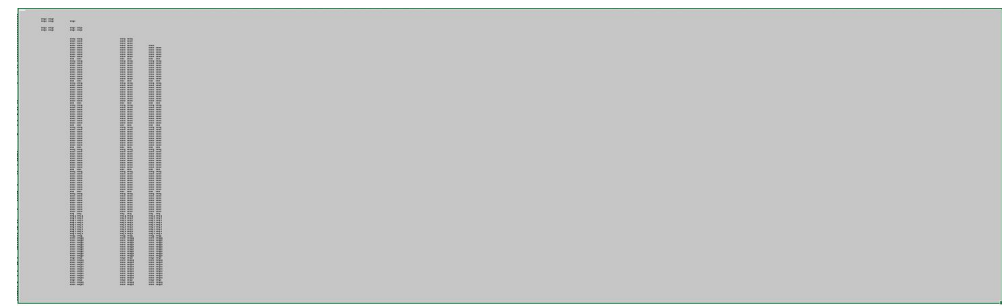

Fig 7.3. This screen shot is for $D F=50$. We see 6 Full column hits and 8 hits.

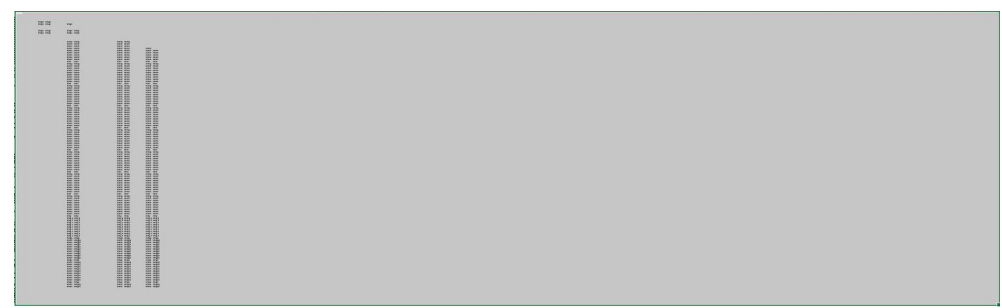

Fig 7.4. This screen shot is for $D F=75$. We see 6 Full column hits and 8 hits.

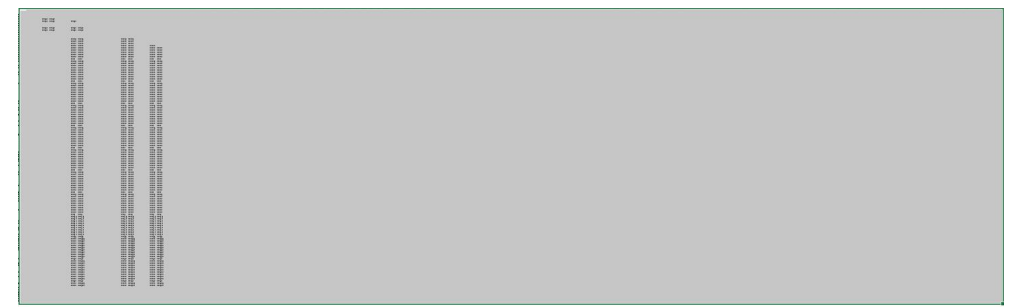


Fig 7.5. This screen shot is for $D F=100$. We see 6 Full column hits and 8 hits.

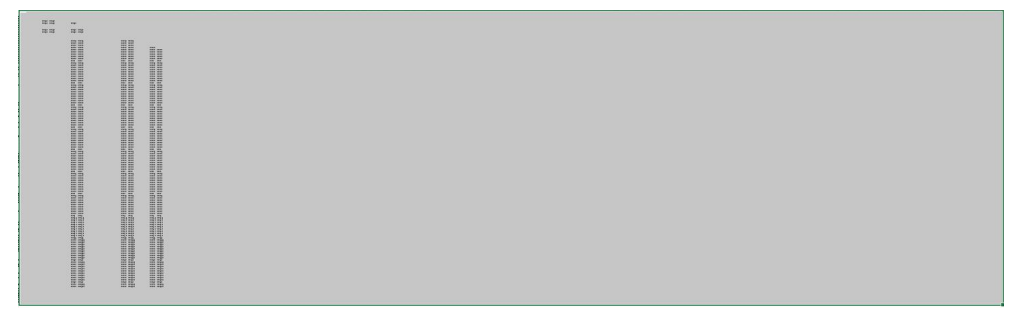

Fig 7.6. This screen shot is for $D F=300$. We see 6 Full column hits and 8 hits.

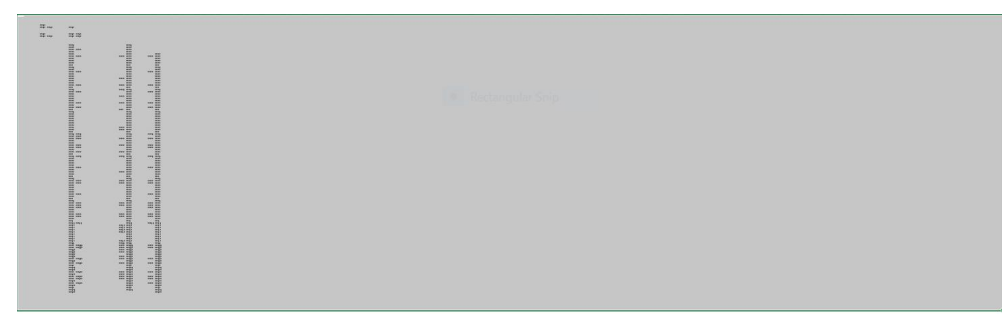

Fig 8.1. This screen shot is for $D F=450$. We see 3 Full column +3 scattered column hits and 6 hits.

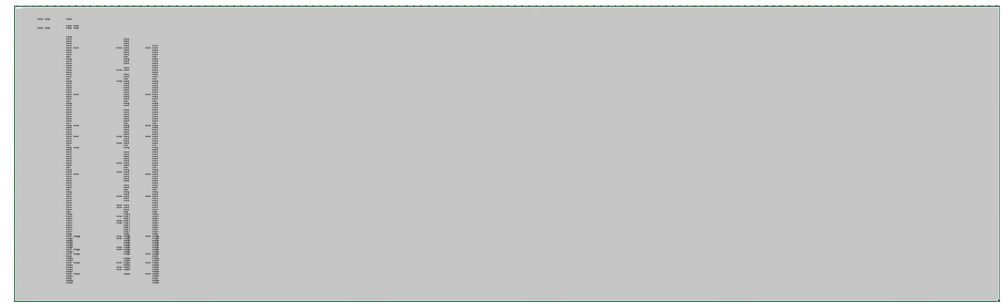

Fig 8.2. This screen shot is for $D F=500$. We see 3 Full column hits +3 scattered column hits and 4 hits.

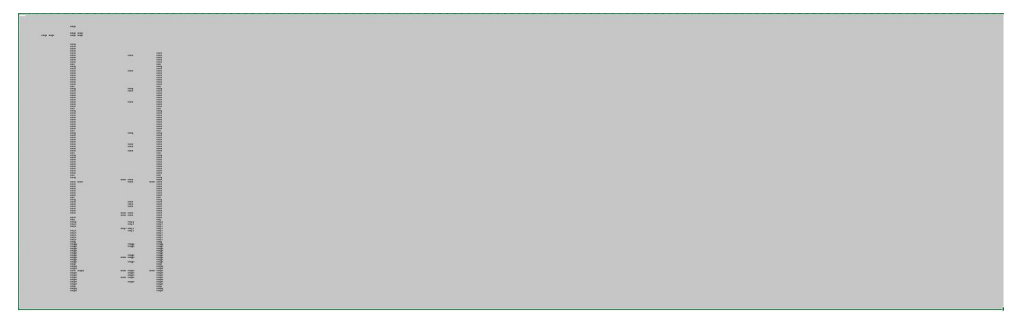

Fig 8.3. This screen shot is for $D F=600$. We see 2 Full column hits +1 scattered column hits and some scattered hits.

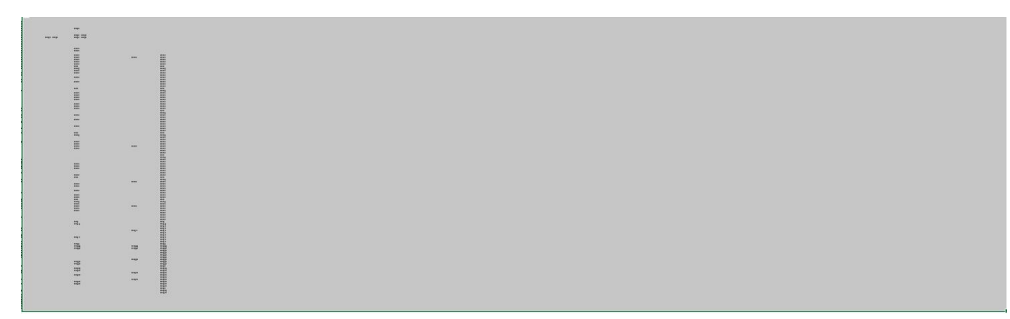

Fig 8.4. This screen shot is for $D F=750$. We see 2 Full column hits +1 column with 10 scattered hits and 4 hits. 


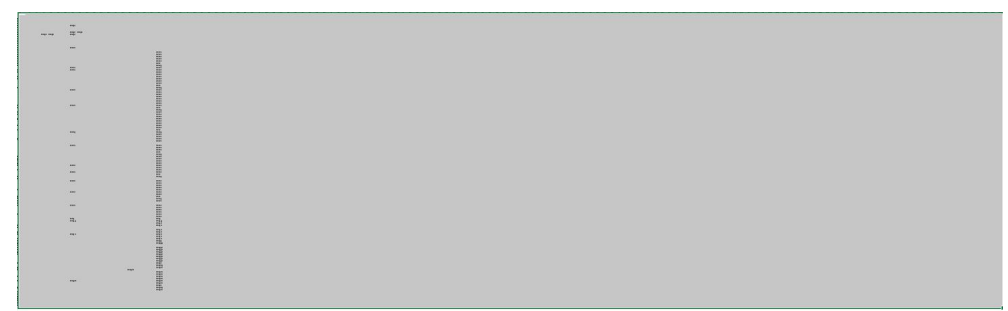

Fig 8.5. This screen shot is for $D F=1000$. We see 1 Full column hits +1 scattered column hits and 2 hits.

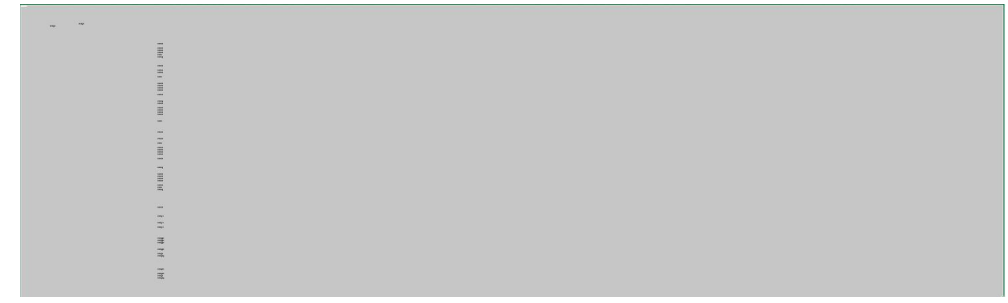

Fig. 9.1. This screen shot is for $D F=3000$. We see 1 scattered column hits and 2 hits.

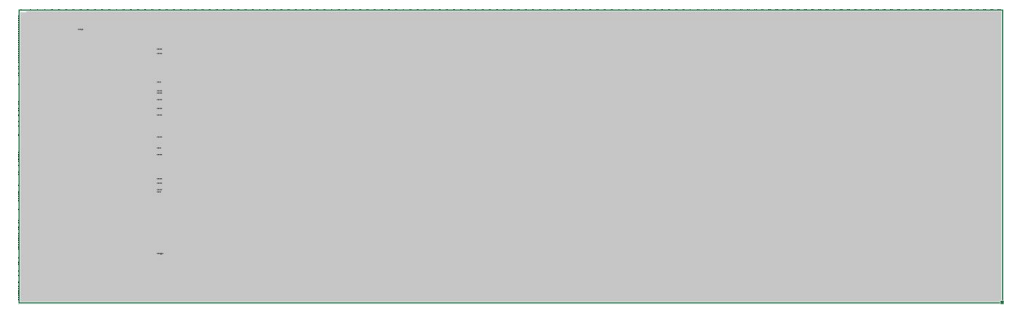

Fig 9.2. This screen shot is for $D F=4500$. We see 1 scattered column hits and 1 hit.

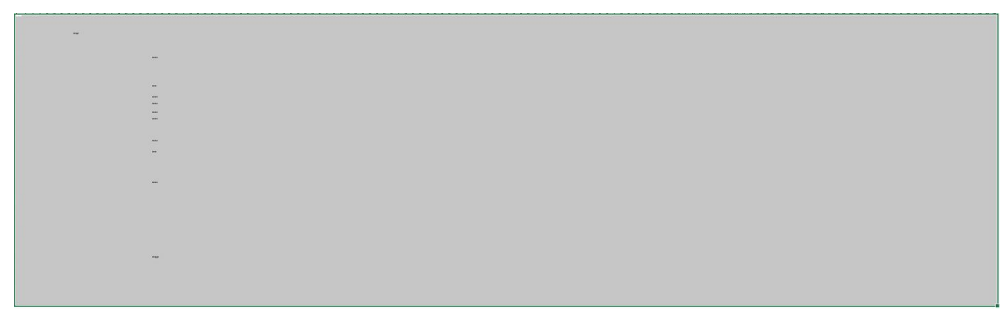

Gig 9.3. This screen shot is for $\mathrm{DF}=\mathbf{5 0 0 0 .}$

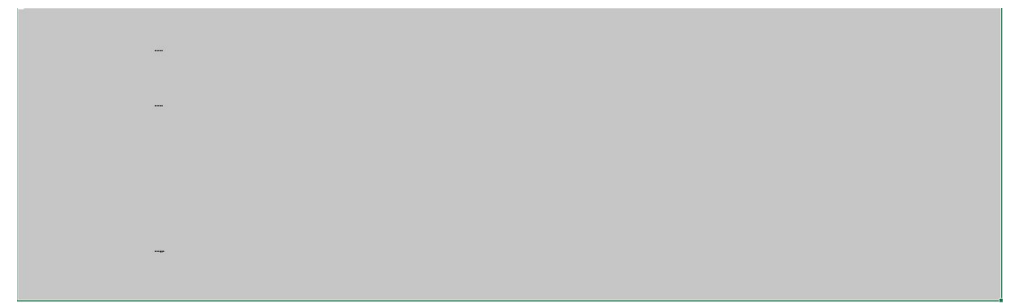

Fig 9.4. This screen shot is for $D F=7500$. We see 1 column of 3 hits. 
Fig 9.5. This screen shot is for $D F=10000$. We see 1 hit.

Fig 10. This screen shot is for $D F=15000$ to $D F=90000$. We see it is totally Blank.

\subsection{Observations on Screen Captures:}

The above screen shots show the different states of $\mathrm{H}_{2} \mathrm{O}$ depending on inter molecular distances. These screen shots were based on different Distance factors (DFs) ranging from 0.02 to 90000 . They show a gradual transition of change of states of $\left(\mathrm{H}_{2} \mathrm{O}\right)$ water as a function of Distance factor. We used the term $\mathrm{H}_{2} \mathrm{O}$ as 'water' is also another state.

We can observe 'Ice' states for DF values equal to 0.02 to 0.08 in Fig 2. Here we see all columns are full of Inter molecular Hits.

The screen captures in Fig 3.1 to Fig 3.6 also show the Ice states for DF values from 0.1 to 0.175. We can observe in first column below 20 are all blanks and bottom-line pitting started. The screen capture in Fig 4.1. to Fig 4.3. show 'Ice + some water' states and we can see ice started melting. We can observe Missing first column of hits and some pitting on overall triangle. In these states first column are all blanks and bottom line, second and third column pitting started. These are for DF values from 0.3 to 0.8 .

The screen capture in Fig 5.1. to Fig 5.4.. are 'water + some ice' states and we can see ice melted. Common factor is ' 8 full columns of hits'. We can also observe Missing first column of hits and with 4 hits or above in next two columns. Pitting in $4^{\text {th }}$ column is increased gradually as well as pitting on the overall triangle. These are for DF values 0.9 to 1.5 .

The screen capture in Fig 6.1. to Fig 6.4. are 'water + much less Ice' states we can see ice melted. Common factor is ' 6 full columns of hits'. We can see water is getting evaporated. Common factor is ' 6 full columns of hits.' We can also observe Missing first two columns of 
hits and with 6 hits or above in next three columns. Pitting on the overall triangle is increased gradually. These are for DF values 3 to 7.5 .

The screen capture in Fig 7.1. to Fig 7.6. are 'water' states we can see water is getting evaporated. Common factor is ' 6 columns of full hits'. We can also observe Missing first two columns of hits and with 6 hits or above in next three columns. Pitting on the overall triangle is increased gradually to full blank after the 6 columns. These are for DF values 9 to 300 .

The screen capture in Fig 8.1. to Fig 8.5. are 'water in evaporation' states. The earlier common factor of ' 6 full columns of hits' was slowly disintegrated and vanished. We can see water is getting evaporated. In other words, pitting on the 6 columns of hits is increased gradually. These are for DF values 450 to 1000 .

The screen capture in Fig 9.1. to 9.5. are 'water fully evaporated' states. The only common factor of 'one column of hits' was slowly disintegrated and vanished. We can see water got getting evaporated fully. These are for DF 5000 to 10000 .

The screen capture in Fig 10. is 'water vapour fully scattered' state for DF values 15000 to 90000. All these screen captures are blank without any hits. The distances between molecules so high, it becomes almost impossible to for any molecule to hit another.

This way these screen captures for DF values 0.02 to 90000 show gradual transmission of states of water as function of inter molecular distances.

\subsection{Full EXCEL file and Data Availability:}

We uploaded a file named 'Vak REF DF 1 hits in 8 col and scattered hits.XLS. ' in Zenodo. with a doi: https://doi.org/10.5281/zenodo.5569547.

\section{SSMMT vs Sita Simulations}

Basic difference between SSMMT and Sita Simulations are the values of masses and distances between the masses. Mathematical frame work is exactly the same. Use the molecular masses and inter molecular distances for SSMMT in SITA simulations this will become SSMMT.

SITA solution can be used for solving presently unsolved problems in many situations like ...

1. prediction of large number of Blue-shifted Galaxies (>5) at Universe Level. 
2. Missing mass due to Star circular velocities and Galaxy disk formation at Galaxy level,

3. Pioneer anomaly at the Solar system level,

4. For conversion of Energy to Matter at Energy level.

SSMMT Simulations

We use SSMMT Simulations for finding the Combined Vector Force using Newtonian Gravitation as Binding Force Between $\mathrm{H}_{2} \mathrm{O}$ Molecules for explaining the Basic Nature of Inter Molecular Attraction Forces and to explain the formation of Three States of Water using SSMMT at Nanoparticle level.

This is totally a new application at $\mathrm{H}_{2} \mathrm{O}$ Molecules level.

\subsection{Discussion}

Finally, Dynamic Universe Model entered the new era and new subject of NANOBIOTECHNOLOGY, which is a budding science and is a conglomeration of four main branches of science viz, Physics, Biology, and chemistry and Engineering.

Assembly of molecules in test tubes usually hinder the behaviors of single molecules. The ensemble averages out the behavior of single molecules. Now with the help of this SSMMT we don't have to do elaborate methods to Isolate single molecule, to do variety of EXPERIMENTS with SIMPLER and CHEAPER LAB equipments. Simple experimental Fluctuations NEED NOT be screened out. As usual the observation of molecular events can be done with single molecule theories.

Mathematics for 'single molecule' theories were fully developed. There are many for problems and difficulties for isolating single molecule for observations via an optical micro scope. With SSMMT such problems will be reduced.

\section{CONCLUSION:}

We can safely conclude the inter molecular forces are the 'sum' of Vector Forces using Newtonian Gravitation attraction as Binding Force Between Molecules or in other words SITA forces. Here in this paper, we used SITA forces for explaining the Basic Nature of Inter Molecular Attraction Forces. 
Now we can safely conclude, Newtonian Gravitation is necessary, appropriate and sufficient for explaining the basic nature of inter molecular attraction forces between $\mathrm{H}_{2} \mathrm{O}$ molecules as defined by Multi Molecule theory. We already know SSMMT uses collective gravitational attraction all the molecules on any single molecule in the collection of molecules.

Earlier researchers found the value of attraction forces but I could not get any work showing the basic nature or reason of attraction of Inter molecular attraction.

\section{ACKNOWLEDGEMENTS:}

I thank Maa Vak for the continuous guidance \& encouragement for solving these totally unknown situations and helping me pass through all the hurdles to attain the victory of solving this problem. I thank God Vishnu for giving the courage to solve this problem.

\section{Data Availability / Data Sharing:}

Satyavarapu Naga Parameshwara Gupta. (2021). Vak REF DF 1 hits in 8 col and scattered hits.XLS. Zenodo. https://doi.org/10.5281/zenodo.5569547

\section{References}

[1] Purcell C: Life at low Reynolds number. Am J Phys 1977, 45:3-11.

[2] Daniel Riveline 'Single molecule': theory and experiments, an introduction From Nanophysics for Health, Journal Of Nanobiotechnology, Mittelwhir, France. 5-9 November 2012Riveline Journal of Nanobiotechnology 2013, 11(Suppl 1):S1 http://www.jnanobiotechnology.com/content/11/S1/S1 Correspondence: riveline@unistra.fr [7]2. Purcell C: Life at low Reynolds number. Am J Phys 1977, 45:311. 6. Schuler B, Eaton WA: Protein folding studied by single-molecule FRET. Curr Opin Structl Biol 2008, 18(1):16-26.

[3] Papai G, Weil PA, Schultz P: New insights into the function of transcription factor TFIID from recent structural studies. Curr Opin Genet Dev 2011, 21(2):219-224.

[4] Meglio A, Praly E, Ding F, Allemand J-F, Bensimon D, Croquette V: Single DNA/protein studies with magnetic traps. Curr Opin Struct Biol 2009, 19(5):615-622. 
[5] Alder, B. J.; Wainwright, T. E. (August 1959). "Studies in Molecular Dynamics. I. General Method". The Journal of Chemical Physics. 31 (2): 459-466. Bibcode:1959JChPh..31..459A. doi:10.1063/1.1730376.

[6] Rahman, A. (19 October 1964). "Correlations in the Motion of Atoms in Liquid Argon". Physical Review. 136 (2A): A405-A411. Bibcode:1964PhRv..136..405R. doi:10.1103/PhysRev.136.A405.

[7] Garrett, Reginald H.; Grisham, Charles M. (2016). Biochemistry (6 ${ }^{\text {th }}$ ed.). University of Virginia. pp. 12-13.

[8] Tschumper, Gregory S. (20 October 2008). "Reliable Electronic Structure Computations for Weak Noncovalent Interactions in Clusters". In Lipkowitz, Kenny B.; Cundari, Thomas R. (eds.). Reviews in Computational Chemistry. 26. John Wiley \& Sons. pp. 3990. doi:10.1002/9780470399545.ch2 (https://doi.org/10.100 2\%2F9780470399545.ch2). ISBN 9780470399545.

[9] Chemical Bonding Ms Shethi and M. Satake

[10] https://en.wikipedia.org/w/index.php?title=Van der Waals force\&oldid=958850259 This page was last edited on 26 May 2020, at 00:57 (UTC).

[11] H. C. Hamaker, Physica, 4(10), 1058-1072 (1937)

[12] F. London, Transactions of the Faraday Society, 33, 8-26 (1937)

[13] R. Tadmor (March 2001). "The London-van der Waals interaction energy between objects of various geometries". Journal of Physics: Condensed Matter. 13 (9): L195L202. Bibcode:2001JPCM...13L.195T (https://ui.adsabs.harvard.edu/abs/2 001JPCM...13L.195T). doi:10.1088/0953-8984/13/9/101 (https://doi.org/10.1088\%2F0953-8984\%2F13\%2F9\%2F101)

[14] Israelachvili J. (1985-2004). Intermolecular and Surface Forces. Academic Press. ISBN 978-0-12-375181-2.

[15] V. A. Parsegian (2006). van der Waals Forces: A Handbook for Biologists, Chemists, Engineers, and Physicists. Cambridge University Press. ISBN 978-0-521-83906-8.

[16] S.N.P.GUPTA, Dynamic Universe Model: A singularity-free N-body problem solution, VDM Publications, Saarbrucken, Germany (2010)

[17] S.N.P.GUPTA, Dynamic Universe Model: SITA singularity free software, VDM Publications, Saarbrucken, Germany ( 2011)

[18] S.N.P.GUPTA, Dynamic Universe Model: SITA software simplified VDM Publications, Saarbrucken, Germany (2011) 
[19] S.N.P.GUPTA, 'Dynamic Universe Model Predicts the Live Trajectory of New Horizons Satellite Going To Pluto' Applied Physics Research, 7(4), pp. 63-77 (2015)

[20] J. V. Narlikar, 'Introduction to cosmology', Foundation books, New Delhi , India (1983)

[21] S.N.P.GUPTA, "Dynamic Universe Model Explains the Variations of Gravitational Deflection Observations of Very-Long-Baseline Interferometry" Applied Physics Research, 6(4), pp.1-16, (2014) https://en.wikipedia.org/w/index.php?title=Van der Waals force\&oldid=958850259 This page was last edited on 26 May 2020, at 00:57 (UTC). 